\title{
Factors influencing IOP changes in postmenopausal women
}

\author{
Chin P. Siuw ${ }^{1}$, Suresh Vasudevan ${ }^{1}$, Mushawiathi Mustapha ${ }^{2}$
}

\section{Abstract}

Objective: Intraocular pressure (IOP) is essential in maintaining normal function of the eye. High IOP is associated with glaucoma. Many physiological factors, including age and hormones, can cause variation in IOP. This study was designed to investigate IOP changes in postmenopausal women and the associated factors, which included sex hormones and body mass index (BMI).

Methods: This was a cross-sectional study. Ninety-eight women were recruited: 49 premenopausal women and 49 postmenopausal women. IOPs between the two groups were compared. The influence of estradiol, progesterone, testosterone, and BMI on IOP was analyzed by a multivariate method. $P<0.05$ was considered significant.

Results: The age of the premenopausal group was $47.14 \pm 3.93$ years and that of the postmenopausal group was 52.10 \pm 3.04 years. The other factors in both groups, which included BMI, blood pressure, and central cornea thickness, were not significantly different. The mean IOP in the postmenopausal group was significantly higher than the mean IOP in the premenopausal group $(15.26 \pm 2.96 \mathrm{~mm} \mathrm{Hg}$ vs. $14.07 \pm 2.65 \mathrm{~mm} \mathrm{Hg}, P=0.04)$. BMI had a weak positive correlation with IOP in premenopausal women $(r=0.31, P=0.03)$. Estradiol was less likely to influence IOP changes in the postmenopausal group as compared with the premenopausal group $(B=-0.021, P=0.002)$. IOP was not significantly related to progesterone and testosterone levels.

Conclusion: Menopausal status has a significant effect on IOP, with a significant increase in IOP seen in postmenopausal women as shown by our study. Estradiol was shown to be a protective factor in reducing IOP among postmenopausal women. Through understanding of the influence of postmenopausal status and sex hormones on IOP, glaucoma management may be improved and the target group for disease screening may be more specific.

Keywords: Intraocular pressure; postmenopausal; hormones; body mass index

Significance statement: Intraocular pressure (IOP) is essential in maintaining normal function of the eye. High IOP is associated with glaucoma. Many physiological factors, including age and hormones, can cause variation in IOP. Menopausal status has a significant effect on IOP, with a significant increase in IOP seen in postmenopausal women as shown by our study. However, changes in IOP with menopausal status do not appear to have any correlation with sex hormone changes, but body mass index had a weak positive correlation with IOP in premenopausal women. Through understanding of the influence of postmenopausal status and body mass index on IOP, glaucoma management may be improved and the target group for disease screening may be more specific.
1. Ophthalmology Department, Sultanah Aminah Hospital, Johor Bahru, Johor, Malaysia

2. Ophthalmology Department, Pusat Perubatan University, Kebangsaan, Jalan Yaacob Latif, Bandar Tun Razak, Wilayah Persekutuan Kuala Lumpur, Malaysia

CORRESPONDING AUTHOR Chin P. Siuw

Ophthalmology Department, Sultanah Aminah Hospital, Jalan Persiaran Abu Bakar Sultan, 80100, Johor Bahru, Johor, Malaysia

Tel.: +607-2257000

E-mail: schinp@yahoo.com

Received 19 December 2017; Accepted 8 March 2018 


\section{Introduction}

Intraocular pressure (IOP) is one factor that can be measured and controlled in glaucoma. Many factors, such as the time of the day, heart rate, respiratory rate, exercise, fluid intake, systemic medications, topical medications, and pregnancy, can influence IOP [1].

Normal physiological processes can also influence IOP. Menopause is recognized after 12 consecutive months of amenorrhea. Serum levels of the hormones estrogen, progesterone, and testosterone have been shown to drop with menopause and aging [2].

Hulsman et al. [3] found an association between early menopause and open-angle glaucoma. Women who went through natural menopause before the age of 45 years had a higher risk of open-angle glaucoma (odd ratio 2.6, 95\% confidence interval 1.5-4.8). Ebeigbe et al. [4] found that IOP in their postmenopausal group was significantly higher than that in their premenopausal group. There was a significant positive correlation between IOP and blood pressure (BP). Studies also documented higher IOP in postmenopausal women and a reduction in IOP with hormone replacement therapy (HRT) [5]. Thus hormonal changes in menopausal women might have an influence on IOP, and the lower the level, the higher the IOP in postmenopausal women.

Globally, glaucoma is the leading cause of irreversible blindness [6]. Raised IOP is one of the factors that can cause glaucoma [7]. The incidence of IOP has been found to significantly increase in postmenopausal women compared with premenopausal women $[4,5,8]$. It was postulated that hormonal changes may have an effect on IOP [5, 8, 9]. Postmenopausal women receiving HRT had a significantly lower mean IOP compared with the non-HRT group [10-13]. However, some studies did not show a significant change in IOP with HRT [9, 14]. Toker et al. [9] found that increased testosterone levels had a positive correlation with changes in IOP ( $r=0.48, P=0.02)$. Other than hormones, body mass index (BMI) and metabolic syndrome have also been associated with changes in IOP in postmenopausal women $[8,15]$. Panchami et al. [8] showed that IOP in their postmenopausal group positively correlated with BMI ( $r=0.381, P=0.002)$.

The objective of this study was to investigate the changes in IOP with menopausal status, and the association with BMI and hormone level changes in premenopausal and postmenopausal women in Malaysia.

\section{Materials and methods \\ Patients and study design}

This is a cross-sectional study conducted from May 2016 to August 2017 at Sultanah Aminah Hospital, Johor Bahru, Johor, Malaysia. Healthy female patients aged between 40 and 55 years attending the Ophthalmology Clinic at Sultanah Aminah Hospital for an eye examination were recruited for this study. Ninety-eight patients were selected by convenient sampling, among which 49 healthy female patients younger than 55 years and who had had amenorrhea for more than 1 year were considered to be the postmenopausal group, and 49 healthy women who had still menstruating for at least the previous 6 months were considered as the premenopausal group.

Female patients with uncontrolled diabetes mellitus, uncontrolled hypertension, chronic renal disease, chronic liver disease, systemic lupus erythematosus, and glaucoma were excluded from this study. Patients who had undergone an ocular operation within the previous 3 months, who had undergone surgical menopause, or who were taking topical/ systemic steroids or hormones were also excluded from the study.

\section{Ophthalmic parameters}

Patients were examined by the same investigator, and IOP measurements were taken by Goldmann applanation tonometry. IOP is determined by the amount of force required to flatten a constant cornea surface over an area with a diameter of $3.06 \mathrm{~mm}$. After fluorescein has been put into the tear film and under viewing with cobalt blue light, a biprism is used to applanate the cornea surface. By rotation of the dial, two mires move. When the inner margins of the two semicircles in the viewfinder just touch, an area of the cornea with a diameter of $3.06 \mathrm{~mm}$ is applanated. IOP is then obtained from the measurement. Two IOP readings were taken, and the average of the readings was recorded. Only the right eye IOP was analyzed in this study.

Central cornea thickness (CCT) was measured with a pachymeter. The participants' weight, height, and diastolic 
and systolic BP were recorded, and BMI was calculated in the unit of kilograms per meter. Serum levels of estradiol, progesterone, and testosterone were measured.

All tests were done between 11 am and noon during a clinic visit to avoid diurnal variation in IOP. This study was approved by the Medical Research and Ethics Committee of Malaysia (NMRR-15-2266-28579). Informed consent was obtained from all patients.

\section{Statistical analysis}

The descriptive statistics are presented as the mean \pm standard deviation. PASW Statistics (version 18) was used to analyze the data. Comparison of IOPs between the premenopausal group and the postmenopausal group was performed with Student's $t$ test. Pearson's correlation coefficient was calculated to find the correlation between BMI and IOP. Multivariable logistic regression was conducted to evaluate the independent associations for each risk factor. Adjusted odds ratios and $95 \%$ confidence intervals are presented. $P<0.05$ was regarded as statistically significant.

\section{Results}

The age of the postmenopausal group was $52.10 \pm 3.036$ years and that of the premenopausal group was $47.14 \pm 3.932$ years. Significant differences were found in IOP, estradiol level, progesterone level, and testosterone level between the premenopausal group and the postmenopausal group $(P<0.05)$. There were no differences in BMI, BP, and CCT between these two groups. The results are given in Table 1 .

Table 2 shows positive correlations between IOP and BMI $(P=0.031)$ and between IOP and CCT $(P=0.002)$ among the premenopausal group. Figures 1 and 2 show both correlations as scatterplots. In the postmenopausal group, no significant correlations were found between IOP and BMI, BP, CCT, and hormones.

Multivariable analysis using multiple logistic regression showed that estradiol is less likely to influence IOP changes in the postmenopausal group as compared with the premenopausal group (Table 3).

\section{Discussion}

IOP was $0.19 \mathrm{~mm} \mathrm{Hg}(P=0.04)$ higher in postmenopausal women than in premenopausal women. The estradiol, progesterone, and testosterone levels were significantly different in premenopausal woman and postmenopausal women; the mean values were $384.35 \mathrm{pmol} / \mathrm{L}$ and $32.73 \mathrm{pmol} / \mathrm{L}(P<0.001)$ for estradiol, $8.84 \mathrm{nmol} / \mathrm{L}$ and $0.39 \mathrm{nmol} / \mathrm{L}(P<0.001)$ for progesterone, and $0.62 \mathrm{nmol} / \mathrm{L}$ and $0.38 \mathrm{nmol} / \mathrm{L}(P=0.004)$ for testosterone. The mean age in the premenopausal group was 47.14 years compared with 52.10 years in the postmenopausal group. There was positive correlations between IOP and BMI $(r=0.308, P=0.031)$ and between IOP and CCT $(r=0.437$, $P=0.002)$ in the premenopausal group. However, in the postmenopausal group, no significant correlations were found

Table 1. Comparison of body mass index (BMI), intraocular pressure (IOP), blood pressure (BP), central cornea thickness (CCT), and hormone levels between the premenopausal group and the postmenopausal group

\begin{tabular}{|c|c|c|c|c|c|c|}
\hline \multirow[t]{2}{*}{ Variable } & \multirow[t]{2}{*}{ Premenopausal } & \multirow[t]{2}{*}{ Postmenopausal } & \multirow[t]{2}{*}{$t$} & \multirow[t]{2}{*}{$P$-value } & \multicolumn{2}{|c|}{ 95\% Confidence leve } \\
\hline & & & & & Lower & Uppe \\
\hline BMI $\left(\mathrm{kg} / \mathrm{m}^{2}\right)$ & $25.36 \pm 5.018$ & $24.49 \pm 4.818$ & 0.876 & 0.383 & -1.103 & 2.843 \\
\hline $\mathrm{IOP}(\mathrm{mm} \mathrm{Hg})$ & $14.07 \pm 2.650$ & $15.26 \pm 2.962$ & -2.085 & 0.040 & -2.311 & -0.057 \\
\hline Systolic BP (mm Hg) & $129.2 \pm 13.097$ & $125.88 \pm 12.639$ & 1.279 & 0.204 & -1.835 & 8.488 \\
\hline Diastolic BP (mm Hg) & $73.14 \pm 0.145$ & $73.18 \pm 10.806$ & -0.202 & 0.984 & -4.055 & 3.973 \\
\hline $\mathrm{CCT}(\mu \mathrm{m})$ & $549.57 \pm 37.770$ & $543.51 \pm 34.421$ & 0.830 & 0.408 & -8.430 & 20.552 \\
\hline Estradiol (pmol/L) & $384.35 \pm 276.006$ & $32.73 \pm 29.150$ & 8.868 & 0.000 & 271.945 & 431.294 \\
\hline Progesterone $(\mathrm{nmol} / \mathrm{L})$ & $8.84 \pm 12.565$ & $0.39 \pm 0.615$ & 4.697 & 0.000 & 4.829 & 12.055 \\
\hline Testosterone (nmol/L) & $0.62 \pm 0.443$ & $0.38 \pm 0.376$ & 2.986 & 0.004 & 0.083 & 0.413 \\
\hline
\end{tabular}

Significant when $P<0.05$. 
Table 2. Correlation between intraocular pressure (IOP) and age, body mass index (BMI), blood pressure (BP), central cornea thickness (CCT), and hormones in the premenopausal group and the postmenopausal group

\begin{tabular}{lrrrr}
\hline Parameter & \multicolumn{2}{c}{ Premenopausal IOP } & \multicolumn{2}{c}{ Postmenopausal IOP } \\
\cline { 2 - 5 } & $r$ & $P$-value & $r$ & 0.157 \\
Age & 0.219 & 0.131 & 0.157 & 0.282 \\
Systolic BP & 0.165 & 0.257 & 0.117 & 0.281 \\
Diastolic BP & 0.100 & 0.493 & -0.056 & 0.423 \\
BMI & 0.308 & 0.031 & 0.143 & 0.326 \\
CCT & 0.437 & 0.002 & -0.016 & 0.911 \\
Estradiol & 0.126 & 0.387 & -0.009 & 0.953 \\
Progesterone & -0.018 & 0.901 & 0.130 & 0.374 \\
Testosterone & 0.178 & 0.221 & & \\
\hline
\end{tabular}

Significant when $P<0.05$.

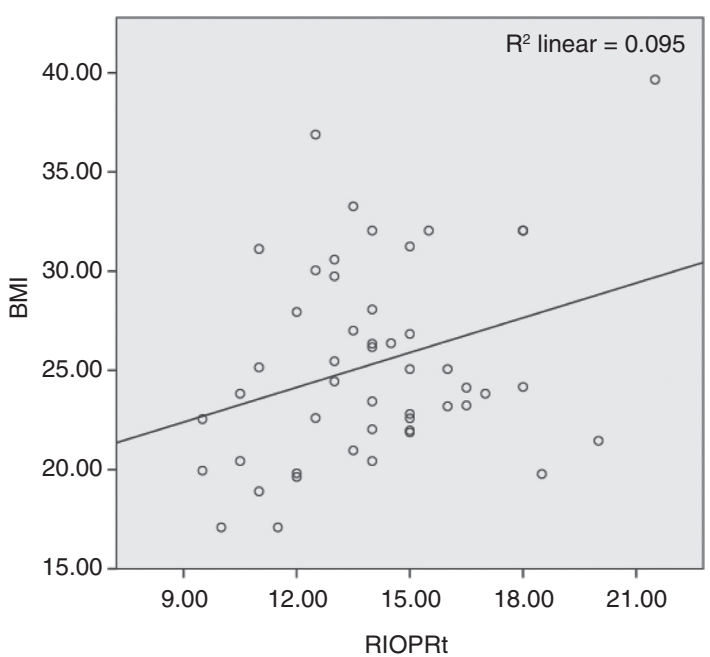

Fig. 1. Scatterplot showing positive correlation between intraocular pressure of the right eye (RIOPRt) and body mass index (BMI) in the premenopausal group.

between IOP and BMI, BP, CCT, and hormone levels. On multivariate analysis, estradiol level was a protective factor in reducing IOP among postmenopausal women $(B=-0.021$, $P=0.02)$.

IOP changes in premenopausal and postmenopausal women had been reported previously, and had been investigated for the association with high BP, metabolic syndrome, BMI, and sex hormone levels [4, 7-9]. In a study of 120 southern Indian females aged 40-55 years, with 60 in the

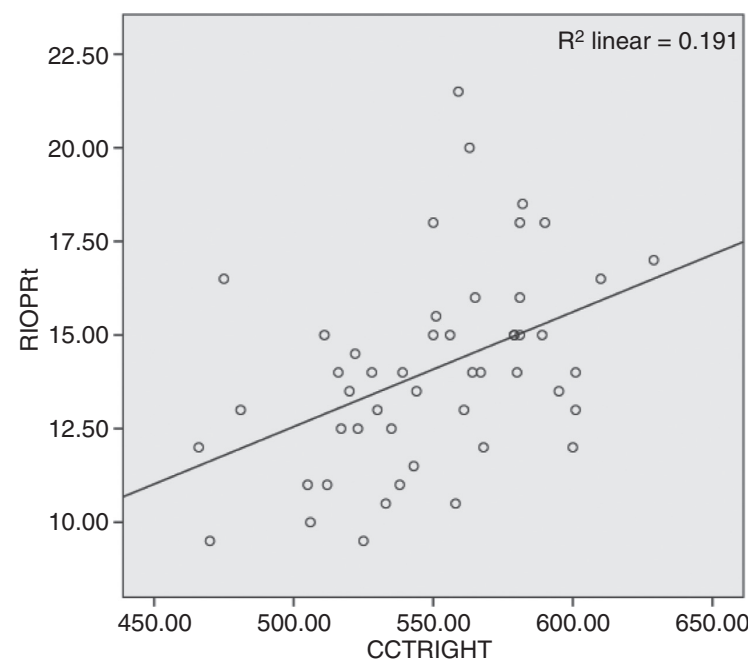

Fig. 2. Scatterplot showing positive correlation between intraocular pressure of the right eye (RIOPRt) and central cornea thickness of the right eye (CCTRIGHT) in the premenopausal group.

premenopausal group and 60 in the postmenopausal group, Panchami et al. [8] reported a $3.24 \mathrm{~mm} \mathrm{Hg}$ higher IOP in the postmenopausal group. The BMI was higher in the postmenopausal group in the same study; a positive correlation with IOP was shown by $r=0.381(P=0.002)$.

In the current study, the postmenopausal group had higher IOP, and the IOPs were within normal limits. The BMI was not significantly different in both groups, but a weak correlation was found only in the premenopausal group. A Shiotz 
Table 3. Factors associated with intraocular pressure (IOP) changes in the premenopausal group and the postmenopausal group

\begin{tabular}{|c|c|c|c|c|c|c|c|}
\hline \multirow[t]{2}{*}{ Variable } & \multirow[t]{2}{*}{ Premenopausal } & \multirow[t]{2}{*}{ Postmenopausal } & \multirow[t]{2}{*}{$B$} & \multirow[t]{2}{*}{$P$-value } & \multirow{2}{*}{$\begin{array}{l}\text { Adjusted } \\
\text { odds ratio }\end{array}$} & \multicolumn{2}{|c|}{$95 \%$ Confidence leve } \\
\hline & & & & & & Lower & Uppe \\
\hline IOP & $14.07 \pm 2.650$ & $15.26 \pm 2.962$ & 0.189 & 0.172 & 1.208 & 0.921 & 1.58 \\
\hline Estradiol & $384.35 \pm 276.006$ & $32.73 \pm 29.150$ & -0.021 & 0.002 & 0.979 & 0.966 & 0.992 \\
\hline Progesterone & $8.84 \pm 12.565$ & $0.39 \pm 0.615$ & -0.808 & 0.214 & 0.446 & 0.125 & 1.595 \\
\hline Testosterone & $0.62 \pm 0.443$ & $0.38 \pm 0.376$ & -0.773 & 0.384 & 0.462 & 0.081 & 2.629 \\
\hline
\end{tabular}

Significant when $P<0.05$.

tonometer was used on patients in the supine position in the study of Panchami et al. [8], while a standard Goldmann applanation tonometer was used in the current study.

Toker et al. [9] assessed the effect of serum hormones on IOP in menopausal women in Turkey. Thirty women receiving HRT and 32 women not receiving HRT were included. The mean IOP was not significantly different in both groups. However, IOP positively correlated with increases in testosterone levels in both groups; $r=0.48$ and $P=0.02$ in women receiving HRT and $r=0.42$ and $P=0.003$ in those not receiving HRT. There was no significant correlation between IOP and estradiol or follicle-stimulating hormone level.

In the current study, the effect of HRT was not investigated; only endogenous estrogen, progesterone, and testosterone were measured. The time of data collection was similar: 10 am-11 am in the study of Toker et al. [9] and 11 am to noon in the current study.

HRT has been associated with lower IOP in postmenopausal women. Tint et al. [10] studied the association between hormone therapy and IOP in nonglaucomatous eyes. Among 263 postmenopausal women, the mean IOP in the hormone therapy group was significantly lower than that in the non-hormonal therapy group, with a difference of $1.41 \mathrm{~mm} \mathrm{Hg}$. Affinito et al. studied 50 postmenopausal women, and found that in 25 of them who were treated with HRT, IOP was significantly lower $(14.1 \pm 2.0 \mathrm{~mm}$ $\mathrm{Hg}$ vs. $16.1 \pm 2.4 \mathrm{~mm} \mathrm{Hg}$, respectively) 3 months after therapy, $P<0.01$ [11]. Six months after therapy, IOP was still significantly lower $(14.0 \pm 2.1 \mathrm{~mm} \mathrm{Hg}$ vs. $16.1 \pm 2.3 \mathrm{~mm} \mathrm{Hg}$, respectively) $P<0.01$. However, both studies involved postmenopausal women who did not have glaucoma. HRT may not have clinical benefit in patients with glaucoma.
Similarly, IOP of 25 white menopausal women without an abnormal ophthalmologic history was measured before and during HRT in a study by Sator et al. [12]. The results showed mean IOP was significantly decreased after 12 weeks of therapy $P<0.001$.

Gauschino et al. [14] studied 80 postmenopausal women aged 52-70 years for 1 year. From comparison of women receiving HRT and women not receiving HRT, no significant changes in IOP were reported.

During pregnancy, increased levels of estrogen have been found to be associated with a $10 \%$ decrease in IOP, and this may be a result of increased outflow facility and decreased venous pressure. The effect of estradiol on regulating vascular resistance and augmenting nitric oxide synthase supports the vascular theory [5]. An increase in nitric oxide levels causes a decrease in IOP by relaxation of the trabecular meshwork [8]. Menopause is associated with reduction in the levels of sex hormones. A decrease in estrogen level may be the main reason for the increase in IOP, in line with the aforementioned theory.

Obesity has been associated with an increase in blood viscosity, and thus increases outflow resistance in episcleral veins, which increases IOP [16].

In the current study, all participants were nonglaucomatous. The mean IOP in this study was within normal limits. No participants received HRT. In the present study, estradiol was a protective factor in significantly reducing IOP among postmenopausal women. Progesterone and testosterone did not significantly influence IOP. This was different from previous studies. Testosterone was correlated with IOP but estradiol and follicle-stimulating hormone did not influence IOP in a previous study [9]. 
In the current study, a standard Goldmann applanation tonometer was used to obtain IOP. IOP was measured and blood was taken for measurement of serum hormone levels at a fixed time of 11 am to noon to minimize diurnal variation.

The participants were from one center. Participants from multiple centers might be better representative of Malaysian society. The participants did not receive HRT, and thus its influence on IOP was not studied. There was only one visit in the study. Long-term follow-up to determine IOP fluctuation in the same participant before and after menopause may provide a clearer picture of the IOP pattern in menopause. Its correlation with weight reduction could also be observed in long-term follow-up of the participants.

Another factor to be considered is age. The age range in the present study was 40-55 years, which was similar to that in a previous study [8]. In the present study, age had no correlation with IOP in both groups. Previous studies showed that IOP increases with age [17]. Wong et al. [18] found that IOP increased with age until the sixth decade, followed by a decrease with a further increase in age. However, IOP was not always found to increase with age. In a cohort study involving 274,064 Korean adults, Zhao et al. [19] found that IOP was inversely associated with age. The average longitudinal change in IOP per 1-year increase was $-0.065 \mathrm{~mm} \mathrm{Hg}(95 \%$ confidence interval -0.068 to $-0.063 \mathrm{~mm} \mathrm{Hg}$ ).

In the present study, CCT was positively correlated with IOP change in the premenopausal group. Tonnu et al. [20] found out the association between IOP and CCT for Goldmann applanation tonometry was a $0.28 \mathrm{~mm} \mathrm{Hg}$ increase in IOP by every $10 \mu \mathrm{m}$ increase in CCT $(P<0.05)$.

In conclusion, the present study indicates that IOP is higher in postmenopausal women. It also demonstrated that estradiol level is a protective factor in significantly reducing IOP among postmenopausal women. BMI was shown to have a positive correlation with IOP changes in the premenopausal group. The results of the study further support an increase in postmenopausal IOP in Asian women. They provide further insight into the influence of BMI and sex hormones on IOP changes in women. Menopause is associated with many factors that need to be evaluated to look for a underlying factor for IOP changes. This would improve our understanding of and further development of new therapy for treating glaucoma.

\section{Acknowledgments}

We thank the Director General of Health, Ministry of Health, Malaysia, for permission to publish this study. We express our gratitude to the Institute of Public Health, Ministry of Health, for its continuous support and technical advice. We also thank Sultanah Aminah Hospital and Pusat Perubatan University Kebangsaan Malaysia for the success of this study. Blood tests were done in the Pathology Department of Sultanah Aminah Hospital, Johor Bahru, Johor, Malaysia.

\section{Conflicts of interest}

The authors declare that they have no conflicts of interest.

\section{Funding}

This research received no specific grant from any funding agency in the public, commercial, or not-for-profit sectors.

\section{Author contributions}

Chin P. Siuw was responsible for conceptualization, literature search, and writing the manuscript. Suresh Vasudevan was responsible for supervising the research study. Mushawiathi Mustapha was responsible for revision of an early draft.

\section{References}

1. Cioffic GA, Durcan FJ, Girkin CA, Gupta N, Piltz-Seymour JR, Samuelson TW, et al. Basic and clinical science course: glaucoma section 10. American Academy of Ophthalmology; 2013 2014. p. 13-26.

2. Manson JE. Chapter 1: overview of menopause. North American Menopause Society; 2010. p. 1.1-1.5. Available from: http:// www.menopause.org/docs/2012/cg_a.pdf\%20Accessed $\% 20$ on\%20May\%2014.

3. Hulsman CA, Westendorp IC, Ramrattan RS, Wolfs RC, Witteman JC, Vingerling JR, et al. Is open-angle glaucoma associated with early menopause? The Rotterdam Study. Am J Epidemiol 2001;154:138-44.

4. Ebeigbe JA, Ebeigbe PN, Ighoroje AD. Intraocular pressure in postmenopausal Nigerian women with and without systemic hypertension. S Afr Optom 2011;70:117-22.

5. Vajaranant TS, Pasquale LR. Estrogen deficiency accelerates aging of the optic nerve. Menopause 2012;19:942-7.

6. Glaucoma is second leading cause of blindness globally. Bull World Health Organ 2004;82:811-90. 
7. Park BJ, Park JO, Kang HT, Lee YJ. Elevated intraocular pressure is associated with metabolic syndrome in postmenopausal women: the Korean National Health and Nutrition Examination Survey. Menopause 2013;20:742-6.

8. Panchami, Pai SR, Shenoy JP, Shivakumar J, Kole SB. Postmenopausal intraocular pressure changes in South Indian females. J Clin Diagn Res 2013;7:1322-4.

9. Toker E, Yenice O, Temel A. Influence of serum levels of sex hormones on intraocular pressure in menopausal women. J Glaucoma 2003; $12: 436-40$

10. Tint NL, Alexander P, Tint KM, Vasileiadis GT, Yeung AM, Azuara-Blanco A. Hormone therapy and intraocular pressure in nonglaucomatous eyes. Menopause 2010;17:157-60.

11. Affinito P, Sardo AD, Carlo CD, Sammartino A, Tommaselli GA, Bifulco G, et al. Effects of hormone replacement therapy on ocular function in menopause. Menopause 2003;10: $482-7$.

12. Sator MO, Joura EA, Frigo P, Kurz C, Metka M, Hommer A, et al. Hormone replacement therapy and intraocular pressure. Maturitas 1997;28:55-8.

13. Sator MO, Akramian J, Joura EA, Nessmann A, Wedrich A, Gruber D, et al. Reduction of intraocular pressure in a glaucoma patient undergoing hormone replacement therapy. Maturitas 1998;29:93-5.
14. Gauschino S, Grimaldi E, Sartore A, Mugittu R, Mangino F, Bortoli $\mathrm{P}$, et al. Visual function in menopause: the role of hormone replacement therapy. Menopause 2003;10:53-7.

15. Pasquale LR. There is more to the relation between intraocular pressure and metabolic syndrome than meets the eye: a connection to estrogen deficiency. Menopause 2013;20:719-20.

16. Bulpitt CJ, Hodes $\mathrm{C}$, Everitt MG. Intraocular pressure and systemic blood pressure in the elderly. Br J Ophthal 1957 59:717-20.

17. Qureshi IA. Age and intraocular pressure: how are they correlated? J Pak Med Assoc 1995;45:150-2.

18. Wong TT, Wong TY, Foster PJ, Crowston JG, Fong CW, Aung T, et al. The relationship of intraocular pressure with age, systolic blood pressure, and central corneal thickness in an Asian population. Invest Ophthalmol Vis Sci 2009;50:4097-102.

19. Zhao D, Kim MH, Pastor-Barriuso R, Chang Y, Ryu S, Zhang Y, et al. A longitudinal study of age-related changes in intraocular pressure: the Kangbuk Samsung Health Study. Invest Ophthalmol Vis Sci 2014;55:6244-50.

20. Tonnu PA, Ho T, Newson T, El Sheikh A, Sharma K, White $\mathrm{E}$, et al. The influence of central corneal thickness and age on intraocular pressure measured by pneumotonometry, no contact tonometry, the tono-pen XL, and Goldmann applanation tonometry. Br J Ophthalmol 2005;89:851-4. 